\title{
ON STALLINGS FIBRATIONS
}

\section{LEE NEUWIRTH}

1. Introduction. Of the various special sorts of knot types in $S^{3}$ which have been studied, one of the more tractable subclasses appears to be the knots with finitely generated (and so by [1] free) commutator subgroups. Stallings was able to show [2] that the complement of a small tubular neighborhood of a member of this subclass admits a fibration over $S^{1}$ with fiber an orientable surface with boundary.

In this note the knot groups are characterized among those groups which are extensions of a free group by an infinite cyclic group. Using the construction of this characterization and the theorem in [3] we obtain a simple proof of Stallings' result.

2. Main theorem. We shall suppose from now on that $G$ is a group which extends the free group $F_{2 n}$ by the infinite cyclic group $Z$. We denote by $t$, an element of $G$ which maps onto a generator of $Z$, and by 0 is meant the trivial group.

THEOREM I. If

(1) $F_{2 n}=[G, G]$,

(2) $G /\{t\}=0$,

(3) $\left[t, \prod_{i=1}^{n}\left[a_{i}, b_{i}\right]\right]=1$ in $G$,

where $a_{i}, b_{i}, i=1,2, \cdots, n$, generate $F_{2 n}$, then if the Poincaré conjecture is true there exists a tame knot $k$ in $S^{3}$, such that $\Pi_{1}\left(S^{3}-k\right) \approx G$.

REMARK. By [1] if a knot $k$ in $S^{3}$ has a free commutator subgroup then the rank of this subgroup is even and condition (3) of Theorem I is satisfied. It is well known that a knot group also satisfies condition (2).

Proof of Theorem I. We shall construct a fiber space whose fundamental group is $G$, and which is the complement of an open tubular neighborhood of a knot in a simply connected 3-manifold.

Let $S$ denote a 2-manifold of genus $n$, with boundary a single curve $l$.

Now $G$ is completely described when the automorphism of $F_{2 n}$ induced by $t$ is described. Denote this automorphism by $\phi$. We shall consider $\phi$ as acting on $\pi_{1}(S)$; then condition (2) implies that $\phi$ leaves a class of loops containing $l$, with some orientation, fixed. By dou-

Received by the editors December 21, 1961 and, in revised form, April 2, 1962. 
bling $S$ along $l$, and applying Nielson's theorem [4] we see that $\phi$ is induced by some autohomeomorphism $\Phi$ of $S .^{1}$

Now by matching the two ends of $I \times S$ according to $\Phi$, one obtains a 3-manifold $M$ (with boundary $T$ a torus) whose fundamental group is $G$. Furthermore, by hypothesis, adjoining the relation $t=1$ to $G$ destroys $G$ completely. Thus by matching the boundary of a solid torus $D^{2} \times S^{1}$ with $T$ so as to kill $t$ in $G$, one may obtain a simply connected manifold, such that $M$ is the complement of a tubular neighborhood of a knot, (an interior point of $D^{2}$ ) $\times S^{1}$, and the theorem is proved.

3. Stallings' theorem. Recall the earlier remark that a knot group with free commutator subgroup satisfies conditions (2) and (3) of Theorem I so that a fiber space $M$ may be constructed as above. Now in [3] it is shown that the group system of a knot in $S^{3}$ with finitely generated commutator subgroup determines the homeomorphism type of the complement. This latter proof essentially depends only on the properties of the mapping from the fundamental group of an orientable surface with boundary lying on a tube about the knot, into the fundamental group of the complement of the tube. These properties are easily found to be satisfied for the fiber of the fiber space $M$ which may be constructed as above, and a homeomorphism may be set up exactly as in [3] between $S^{3}$-(neighborhood of $k$ ) and the fiber space $M$. Thus one may bypass the Poincaré conjecture and obtain:

Theorem (Stallings). If a knot, $k$, in $S^{3}$ has a finitely generated commutator subgroup, then $S^{3}$-(tubular neighborhood of $k$ ) is a fiber space over $S^{1}$, with fiber an orientable 2-manifold with boundary.

\section{BIBLIOGRAPHY}

1. L. Neuwirth, The algebraic determination of the genus of knots, Amer. J. Math. 82 (1960), 791-798.

2. J. Stallings, On fibring certain 3-manifolds, University of Georgia Conference on Topology of Manifolds, 1961.

3. L. Neuwirth, The algebraic determination of the topological type of the complement of a knot, Proc. Amer. Math. Soc. 12 (1961), 904-906.

4. J. Nielson, Untersuchungen zur Topologie der geschlossen zweiseiteigen Flächen. I, Acta Math. 50 (1927), 266, Satz 11.

Institute for Defense ANALyses

1 Nielson's theorem states that any automorphism of the fundamental group of a closed orientable 2-manifold is induced by an autohomeomorphism of the 2-manifold. 